\title{
Characterization of Thin Film Displacements in the Electron Microscope
}

\author{
Hidetaka Sawada ${ }^{1,2,3^{*}}$, Reiner Ramlau ${ }^{4}$, Christopher S. Allen ${ }^{1,2}$ and Angus I. Kirkland ${ }^{1,2}$
}

${ }^{1}$ Department of Materials, University of Oxford, Parks Road, Oxford OX1 3PH, UK

${ }^{2}$ Electron Physical Sciences Imaging Centre (ePSIC), Diamond Light Source Ltd, Didcot, Oxford, OX11 0DE, UK

${ }^{3}$ JEOL UK Ltd., Silver Court Watchmead Welwyn Garden City, Herts., AL7 1LT, UK

${ }^{4}$ Max-Planck-Institut für Chemische Physik fester Stoffe, Nöthnitzer Straße 40, D-01187 Dresden, Germany

*To whom correspondence should be addressed. E-mail: hidetaka.sawada@jeoluk.com

[Key words] Vibrations, Resolution, Thin films

$[\mathrm{Tel}]+44(0) 1707377117[\mathrm{Fax}]+44(0) 1707373254$

\begin{abstract}
It is important to characterize behavior along the normal (z) direction to the plane of a thin film to enable three-dimensional reconstruction at atomic-resolution. In this study, we have investigated displacements of a specimen along the $\mathrm{z}$ direction using dark field HAADF STEM images of single atoms as a function of specimen tilt. These showed an elongation perpendicular to the tilt axis. Experimental measurements of the contrast of single atoms indicates fluctuations in the film along the $\mathrm{z}$ direction with a displacement amplitude of $0.6 \mathrm{~nm}$ (FWHM).
\end{abstract}

Due to aberration correction, modern scanning transmission / transmission electron microscopes (STEM/TEM) [1-4] are capable of lateral resolutions less than $50 \mathrm{pm}$ [5-7]. Furthermore, experimental lateral positions perpendicular to the optic axis have been measured with a precision of a few pm [8-10]. However, the resolution in the direction normal to the plane (z) of a thin film is of the order of only a few $\mathrm{nm}$ as determined by experimental measurement using confocal imaging of extended objects [11] or in high angle annular dark field (HAADF) STEM images of point like objects [12]. Improving the resolution in the z direction in STEM / TEM data is therefore desirable for three-dimensional reconstruction of specimens at the atomic level. Two factors contribute to the resolution along the $\mathrm{z}$ direction being an order of magnitude greater than most atomic spacings. The first is the defocus spread arising from the effects of chromatic aberration, the spread in energy of the source and the stability of the objective lens. The second is the geometrical limitation imposed by a finite convergence angle. These factors are all experimentally measurable and limited by the present electron-optical performance of the instrument. However they can be overcame by correctors for chromatic $[3,13]$ and higher-order geometrical aberrations [1416], as well as improvement of overall instrument stability. The resolution along the $\mathrm{z}$ direction can also be limited by the specimen itself. Although there have been reports showing fluctuations introduced by electron illumination during observations of biological specimens [17, 18], and spatial distribution of vibrational excitations investigated by EELS spectra [19], the motion of a specimen along the $\mathrm{z}$ direction has not yet been quantitatively characterized. In this study we have 
investigated the behavior of a specimen at the atomic level and have measured the displacement of a thin film along the $\mathrm{z}$ direction.

To investigate the specimen behavior along the $\mathrm{z}$ direction, high resolution aberration corrected TEM images of gold particles on a thin carbon film were recorded as a function of specimen tilt (Fig.1) at room temperature. The microscope was operated at an accelerating voltage of $300 \mathrm{kV}$ using a JEM-ARM300F. When the specimen is tilted, displacements in the $\mathrm{z}$ direction are observed as a lateral displacement component, resulting in an anisotropic spatial information limit in a TEM image (Figs.1(b) and (e)). For reference, an image without specimen tilt (Figs.1(a) and (d)) was also recorded using a high tilt holder [Supplementary Material 1]. Spatial information in the diffractogram calculated from an image recorded without a specimen tilt extends to high frequency and there is no anisotropic information limit (Fig.1(d)). However, the diffractogram from an image recorded with a specimen tilt of $40^{\circ}$ along the $x$ axis (Fig. 1(e)) shows an anisotropic information limit with the direction of lower resolution perpendicular to the tilting axis (Fig. 1(b)). The amplitude of this information limit becomes larger for higher tilt angles in both the positive and negative tilt direction [Supplementary Material 2]. For a specimen tilt along the y axis, the direction of the lower information limit is rotated by $90^{\circ}$ but remains perpendicular to the tilt axis [Supplementary Material 2 and 3 for TEM and STEM, respectively]. In contrast, high-resolution images of a rigid crystalline specimen show no anisotropic information limit as a function of specimen tilt [Supplementary Material 4]. These experimental observations indicate that a thin amorphous carbon film is vibrating in the direction normal to the film plane (Fig.1(c)). Similar observations of the film at the edge of a grid square give less pronounced anisotropy in the information limit with tilt [Supplementary Material 5]. This is consistent with the vibrations at positions close to the grid being suppressed due to mechanical support by the rigid frame of the grid.

The amplitude of this vibration was estimated from the value of the anisotropic information limit with specimen tilt in TEM. To estimate the effect of the vibration along the $\mathrm{z}$ direction, the low-pass-filtered diffractogram with specimen tilt was divided by the low-pass-filtered diffractogram pattern without tilt [Supplementary Material 6]. The resultant quotient is equivalent to an envelope function that describes the resolution reduction ratio due to vibration. Fig. 1 (f) shows the line profiles of this quotient along the lower resolution direction for specimen tilts of 20,30 , and $40^{\circ}$. We define a lateral spatial frequency $g_{0.5}=d_{0.5}^{-1}$, which gives a reduction ratio $=0.5(\mathrm{FWHM})$ in the intensity of the diffractogram due to a vibration, assuming that the original diffractogram is unity for all frequency (Fig.1(f)). Hence, $d_{0.5}$ (the lateral information limit) becomes larger with the absolute value of specimen tilt (Table 1). By converting the lateral spatial frequency $g=d^{-1}$ to a vertical spatial frequency in a film through $g_{(\mathrm{z})}=\mathrm{z}_{\mathrm{V}}^{-1}=(d / \sin \theta)^{-1}$, where $\theta$ denotes the specimen tilt angle, the amplitude of displacement along the $\mathrm{z}$ direction in the film can be estimated. A diffractogram calculated from an image of a sinusoidally oscillating specimen in the lateral direction can be described by the square of a Bessel function of zero order $J_{0}{ }^{2}\left(2 \pi A_{J} g_{(\mathrm{z})}\right)$, where $g$ denotes a spatial frequency and $A_{J}$ is an amplitude of vibration [20]. Using the measurement value of $g_{(\mathrm{z}) 0.5}=$ $\mathrm{z}_{\mathrm{v} 0.5}^{-1}$, where $J_{0}^{2}\left(2 \pi A_{J} g_{(z) 0.5}\right)=0.5$, values of lateral displacement, $2 A_{J}$ are calculated for specimen tilts of 20,30 , and $40^{\circ}$ (Table 1). The mean value of $2 A_{J}$ is $0.44 \pm 0.4 \mathrm{~nm}$. However, our STEM observations (see Fig.2) suggest that the specimen oscillation does not follow a simple sinusoidal form and is better described with the function having distribution around the center of the vibration. Thus, the square of a sinc function $\operatorname{sinc}^{2}\left(2 \pi A_{s} g_{(z)}\right)$ was calculated from the value of $\operatorname{sinc}^{2}\left(2 \pi A_{s} g_{(z) 0.5}\right)=0.5 . \quad A_{s}$ corresponds to the amplitude of vibration along the $\mathrm{z}$ direction for the rectangular distribution of the oscillation [20]. The mean value of $2 A_{s}$ is $0.54 \pm 0.05 \mathrm{~nm}$. For a further accurate determination of amplitude of displacement from analysis of experimental diffractograms, the vibration mode and the probability distribution along the $\mathrm{z}$ direction should be considered. 
$<$ Table 1 near here>

Next, we have evaluated the displacement in the $\mathrm{z}$ direction by measuring the movement of an object in real space. For this purpose, single gold atoms on a carbon film were imaged using HAADF STEM in a JEM-ARM300F fitted with a STEM aberration corrector at room temperature. The specimen was prepared by evaporating gold on a carbon film. The thickness of the specimen including gold particles was measured to be $9 \mathrm{~nm}$ using STEM EELS [Supplementary Material 9]. An accelerating voltage of $80 \mathrm{kV}$ and a fast scan of $3 \mu \mathrm{s} /$ pixel with a scan resolution of $9.5 \mathrm{pm} /$ pix over an area of $2048 \times 2048$ pixels was used to reduce the lateral movement of the single atoms, induced by electron irradiation. The convergence semiangle was $25 \mathrm{mrad}$ with a current of $25.4 \mathrm{pA}$ and a detector acceptance angle from $68 \mathrm{mrad}$ to $200 \mathrm{mrad}$. For observations of single atoms using HAADF STEM, the in-focus defocus image can be selected for evaluation as the sharpest image with highest intensity at the position of the atom. Fig. 2(a) shows a high-resolution dark field image of single gold atoms surrounding polycrystalline gold nano-particles without specimen tilt. Under this condition the image of a single gold atom is a sharp symmetric white dot. However, when the specimen was tilted along the $\mathrm{x}$-tilt axis by $45^{\circ}$, the images of single atoms were elongated perpendicular to the x-tilt axis (Fig.2(b)). This elongation can be understood as an effect of film vibration perpendicular to the film plane; the elongated image contrast indicates that the film vibrates along the $\mathrm{z}$ direction and a HAADF STEM image contains information about the lateral component of this vibration, as illustrated in Fig.2(c). In order to analyze this elongation, several sub-field images of isolated atoms at the in-focus position were extracted from HAADF STEM images with specimen tilts from $-45^{\circ}$ to $+45^{\circ}$ with an increment of $5^{\circ}$, and averaged as shown in the upper row in Figs.2(d) and (e) respectively. Low-pass filtered images of the averaged image and fitted Gaussian line profiles are shown in the middle and lower rows of Figs. 2(d) and (e). It is evident that the image blur of single atoms in the HAADF STEM images becomes longer in the direction perpendicular to the $\mathrm{x}$-axis when the tilt angle increases. This is consistent with the lateral component being a projection, $\mathrm{Dv}=z_{v} \cdot \sin \theta$ (Fig.2(c)) which increases with higher tilt angles for a constant amplitude of vibration along the $\mathrm{z}$ direction.

$<$ Figure 2 near here>

Finally, the displacement along the $\mathrm{z}$ direction was estimated quantitatively using HAADF STEM images of single atoms shown in figure 2. For aberration corrected HAADF STEM images, the image of a single atom is convolved with the Gaussian probe size, the width of the atomic potential and the effect of any uncorrected chromatic aberration. To distinguish the vibration component from these effects, each Gaussian function in Figs.2(d) and (e) was de-convolved using the probe function of the $0^{\circ}$ image as $\exp \left(-x^{2} /\left(2\left(s-s_{0}\right)\right)\right)$, where $\mathrm{s}$ is the standard deviation of each Gaussian function and $\mathrm{s}_{0}$ is measured from the $0^{\circ}$ image. Figs. $3(\mathrm{a})$ and (b) show the de-convolved Gaussian functions for each specimen tilt. The FWHM of the de-convolved Gaussian functions corresponds to the lateral image displacement which in turn is equal to the projected component, ( $\left.\mathrm{D}_{\mathrm{v} 0.5}\right)$ of the $\mathrm{z}$ direction displacement. A plot of $\mathrm{D}_{\mathrm{v} 0.5}$ as a function of tilting angle is shown in Fig. 3(c). Fitting to the function, $\mathrm{D}_{V 0.5}=\mathrm{z}_{V 0.5} \cdot \sin \left(\theta-\theta_{0}\right)$, where $\theta$ is the tilt angle and $\theta_{0}$ a tilt offset gives a value for the amplitude of the out of plane displacement of the thin film of $\mathrm{zv} 0.5=0.60 \pm 0.15 \mathrm{~nm}$. 
We find that the amplitude of the vibration largely depends on the details of the specimen [see Supplementary Material 6, 7 and 8]. This indicates that the observed $\mathrm{z}$ displacement originates from the physical properties of the film itself, not from an artifact of the experiment nor a limitation of the instrument (e.g. specimen holder). We now briefly discuss the potential origins of the observed $\mathrm{z}$ displacement. Electron irradiation, thermal energy of the film and beam induced charging are all candidates for the origin of the $\mathrm{z}$ displacement as each can cause vibrations in the film. Determining the frequency of the vibration is key to understanding whether it is thermal (due to ambient temperature or electron beam induced heating) or electrostatic (due to beam induced charging) in origin. In a HAADF STEM image, if the frequency of a vibration is comparable or slower than to the dwell time of the scan, a periodic shift in the image would be expected. In this study, the HAADF STEM images were acquired with a dwell time of $3 \mu \mathrm{s} /$ pixel. However, even at this high scan speed periodic movements in the image were not observed (see for example Fig. 2). This indicates that the frequency of the vibration is greater than $\sim 150 \mathrm{kHz}$. Since the vibration phenomena in TEM were consistent with that in STEM [see, Supplementary Material 2, and 3], behavior of the vibration is independent of the current of the illumination, indicating that thermal energy of the film could be the origin of the $\mathrm{z}$ displacement. Further investigation into the physical origin of these vibrations is ongoing.

We have reported the measurement of the out of plane vibrations in a thin film from analysis of HAADF STEM images of single atoms. The FWHM of vibrations perpendicular to the plane of a thin carbon film was determined to be $0.6 \mathrm{~nm}$. These results answer questions pertaining to the loss of resolution in tilted STEM/TEM images and represent a technique for studying the fundamental mechanics of suspended thin films with sub nanometer precision.

\section{Supplementary Material}

Specimen holders and tilt rotation relationships; TEM Spatial resolution limits with specimen tilt for a carbon film with gold particles; HAADF Spatial resolution limits with specimen tilt for a carbon film with gold particles; HAADF STEM resolution as a function of specimen tilt using a rigid crystalline specimen; Dependence of the information limit on specimen position; TEM estimation of the amplitude of displacements along the $\mathrm{z}$ direction; Dependence of the information limit on specimens; Grid size of the specimen; Specimen details; Tilt angle calibration.

\section{Acknowledgment}

The author thanks to Dr. K. Borisenko of University of Oxford and Dr. S. Morishita of JEOL Ltd. for discussion about the vibration of a specimen.

\section{References}

${ }^{1}$ M. Haider, S. Uhlemann, E. Schwan, H. Rose, B. Kabius, and K. Urban, Nature 392, 768 (1998).

${ }^{2}$ O. L. Krivanek, N. Dellby, and A. R. Lupini, Ultramicroscopy 78, 1 (1999).

${ }^{3}$ F. Hosokawa, H. Sawada, Y. Kondo, K. Takayanagi, and K. Suenaga, Microscopy 62, 23 (2013).

${ }^{4}$ H. Sawada, T. Sasaki, F. Hosokawa, S. Yuasa, M. Terao, M. Kawazoe, T. Nakamichi, T. Kaneyama, Y. Kondo, K.

Kimoto, and K. Suenaga, Ultramicroscopy 110, 958 (2010).

${ }^{5}$ R. Erni, M. D.Rossell, C. Kisielowski, and U. Dahmen, Physical Review Letters 102, 096101 (2009).

${ }^{6}$ H. Sawada, Y. Tanishiro, N. Ohashi, T. Tomita, F. Hosokawa, T. Kaneyama, Y. Kondo, and K. Takayanagi, J. Electron Microsc. 58, 357 (2009).

${ }^{7}$ H. Sawada, N. Shimura, F. Hosokawa, N. Shibata, and Y. Ikuhara, Microscopy 64, 213 (2015).

${ }^{8}$ A. B. Yankovich, B. Berkels, W. Dahmen, P. Binev, and P. M. Voyles, Nature Communications 5, 4155 (2014). 
${ }^{9}$ K. Kimoto, T. Asaka, X. Yu, T. Nagai, Y. Matsui, and K. Ishizuka, Ultramicroscopy 110, 778 (2010).

${ }^{10}$ K. Aso, K. Shigematsu, T. Yamamoto, and S. Matsumura., Microscopy 65, 391 (2016).

${ }^{11}$ P. Wang, G. Behan, M. Takeguchi, A. Hashimoto, K. Mitsuishi, M. Shimojo, A. I. Kirkland, and P. D. Nellist, Physical Review Letters 104, 200801 (2010).

${ }^{12}$ K. Benthem, A. R. Lupini, M. P. Oxley, S. D. Findlay, L. J. Allen, and S. J. Pennycook, Ultramicroscopy 106, 1062 (2006).

${ }^{13}$ M. Haider, H. Muller, S. Uhlemann, J. Zach, U. Loebau, and R. Hoeschen, Ultramicroscopy 108, 167 (2008).

${ }^{14}$ H. Müller, S. Uhlemann, P. Hartel, and M. Haider, Microsc. Microanal. 12, 442, (2006).

${ }^{15}$ I. Maßmann, S. Uhemann, H. Müller, P. Hartel, J. Zach, M. Haider, Y. Taniguchi, D. Hoyle, and R. Herring, Microsc. Microanal. 17, Suppl. 1270 (2011).

${ }^{16}$ H. Sawada, T. Sasaki, F. Hosokawa, S. Yuasa, M. Terao, M. Kawazoe, T. Nakamichi, T. Kaneyama, Y. Kondo, K. Kimoto, and K. Suenaga, J. Electron Microsc. 58, 341 (2009).

${ }^{17}$ M. G. Campbell, A. Cheng, A. F. Brilot, A. Moeller, D. Lyumkis, D. Veesler, J. Pan, S. C. Harrison, C. S. Potter, B. Carragher, and N. Grigorieff, Structure 20, 1823 (2012).

${ }^{18}$ A. F. Brilot, , J. Z. Chen, A. Cheng, J. Pan, S. C. Harrison, C. S. Potter, B. Carragher, R. Henderson, and N. Grigorieff, $J$ Struct Biol. 177, 630 (2012).

${ }^{19}$ M.J. Lagos, A. Trügler, U. Hohenester, and P. E. Batson, Nature 543, 529 (2017).

${ }^{20} \mathrm{~J}$. Frank, Optik 30, 171 (1969). 
Table 1. Amplitude of the lateral information limit $\left(d_{0.5}[\mathrm{~nm}]\right)$ and the amplitude of the displacement along the $\mathrm{z}$ direction Parentheses show the error in the measurement.

\begin{tabular}{|c|c|c|c|c|c|}
\hline Tilt angle $\left[{ }^{\circ}\right]$ & $\sin \theta$ & $\begin{array}{c}d_{0.5}[\mathrm{~nm}] \\
(\text { error }[\mathrm{nm}])\end{array}$ & $\begin{array}{c}\mathrm{Z}_{\mathrm{v} 0.5}[\mathrm{~nm}] \\
(\text { error }[\mathrm{nm}])\end{array}$ & $\begin{array}{c}2 A_{J}[\mathrm{~nm}] \\
(\text { error }[\mathrm{nm}])\end{array}$ & $\begin{array}{c}2 A_{S}[\mathrm{~nm}] \\
(\text { error }[\mathrm{nm}])\end{array}$ \\
\hline 20 & 0.342 & $0.44( \pm 0.11)$ & $1.29( \pm 0.33)$ & $0.46( \pm 0.06)$ & $0.57( \pm 0.08)$ \\
\hline 30 & 0.500 & $0.58( \pm 0.08)$ & $1.16( \pm 0.16)$ & $0.42( \pm 0.03)$ & $0.51( \pm 0.05)$ \\
\hline 40 & 0.643 & $0.78( \pm 0.06)$ & $1.21( \pm 0.10)$ & $0.43( \pm 0.02)$ & $0.54( \pm 0.02)$ \\
\hline
\end{tabular}

Avg. $0.44( \pm 0.04) \quad$ Avg. $0.54( \pm 0.05)$ 
(a)

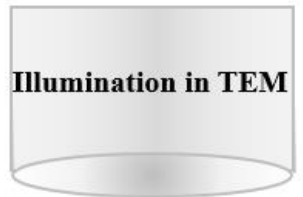

Specimen
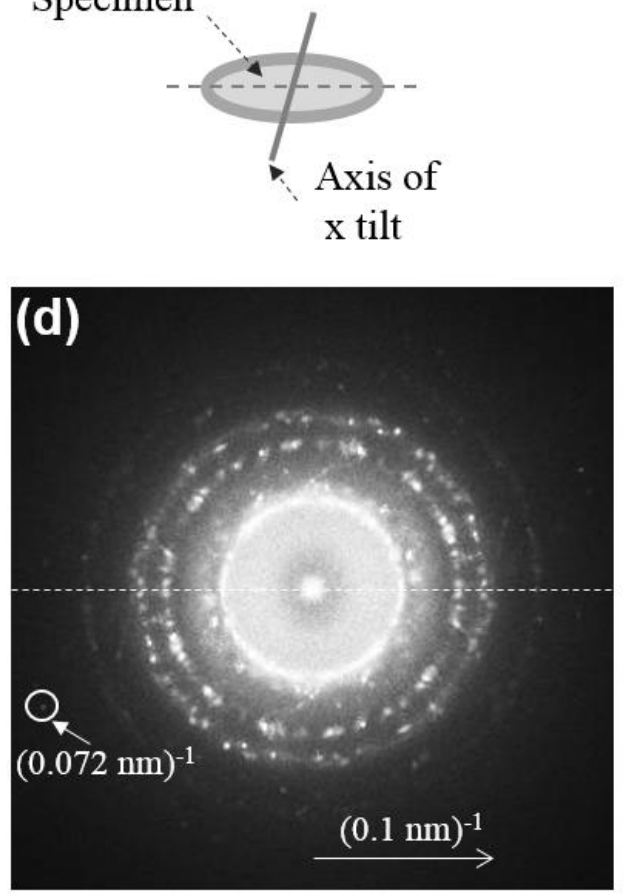

(b)
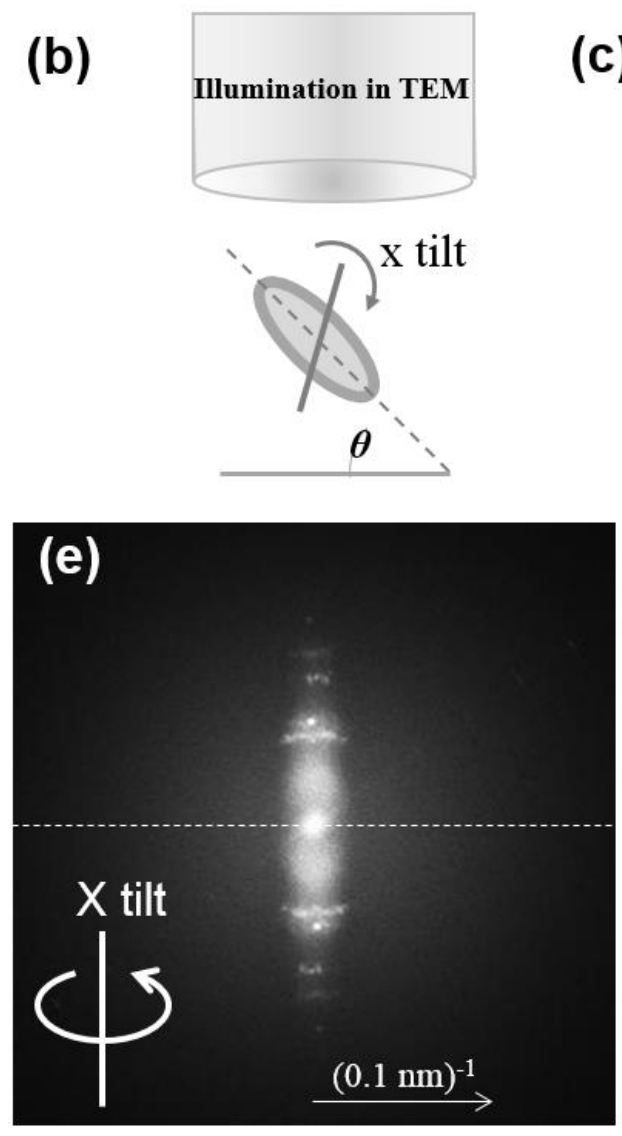

(c)

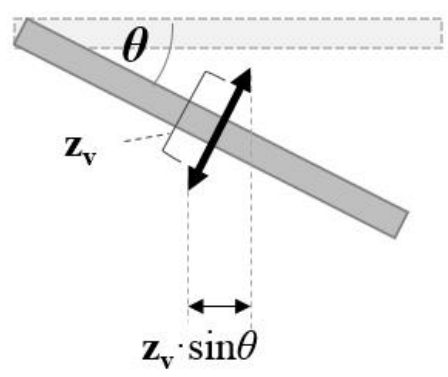

(f)

Reduction ratio by the

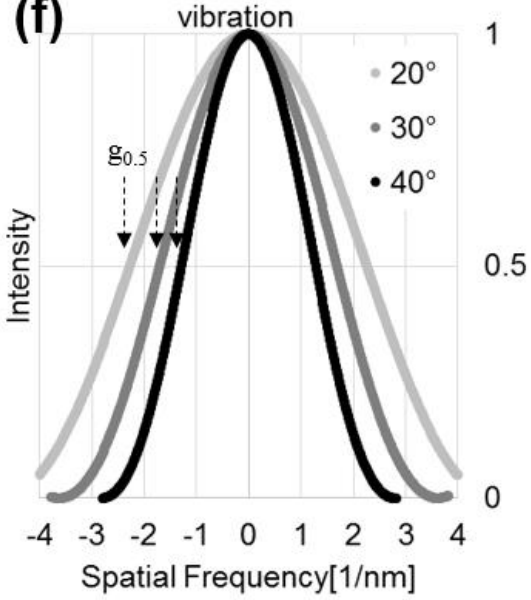

Fig. 1. Schematic of the specimen (a) without tilt and (b) with tilt (b) along the goniometer x axis. (c) Schematic of the relationship between specimen tilt and lateral displacement caused by displacements in the z direction. (d) Diffractogram from TEM images of gold particles on a thin carbon film (Fig.S8(b) in Supplementary Material 8) without specimen tilt calculated from an image recorded at $0^{\circ}$. (e) Diffractogram calculated from an image recorded at $40^{\circ}$ specimen tilt along the $\mathrm{x}$ axis. In (d) and (e) a magnification of $600 \mathrm{kX}$ was used with an acquisition time of 0.6s. (f) Quotients extracted from diffractograms as described in the text along the direction of the lower information limit for images recorded at tilts of 20,30, and $40^{\circ}$. 

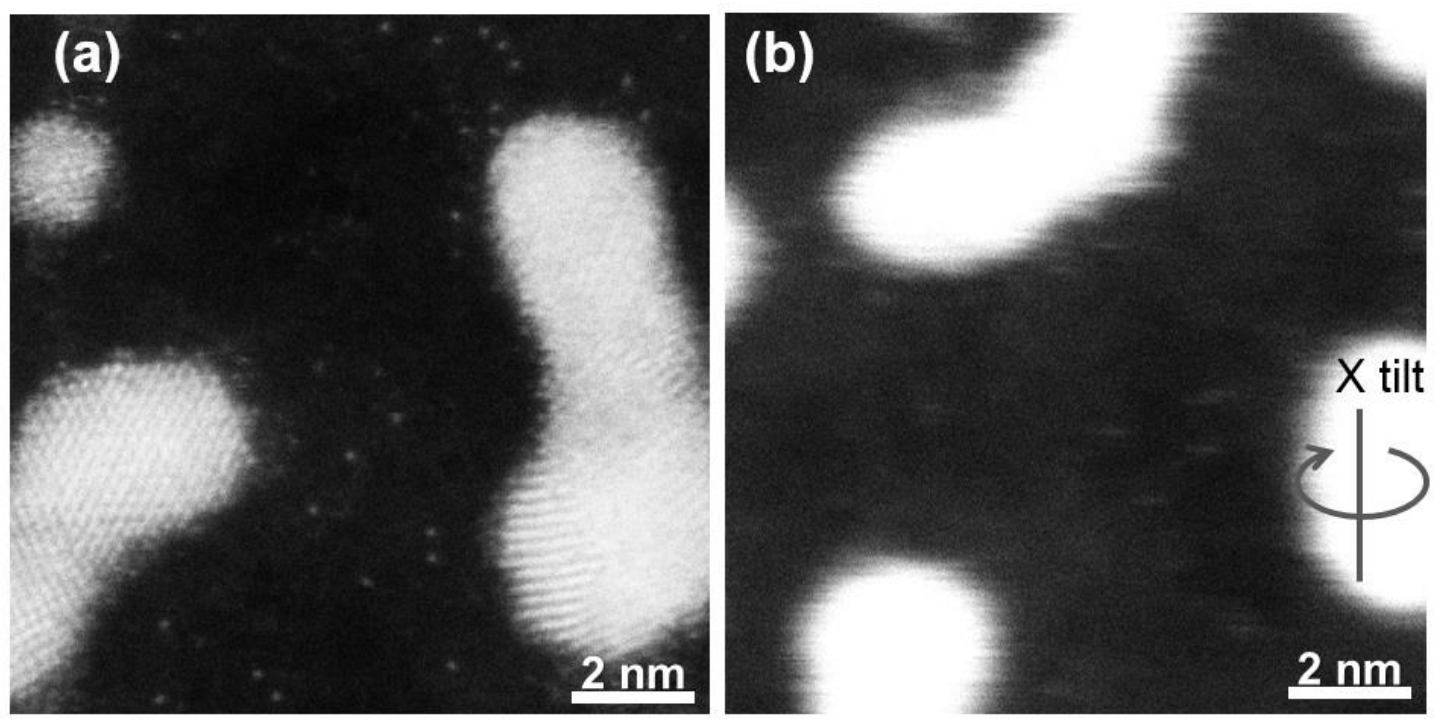

(c)

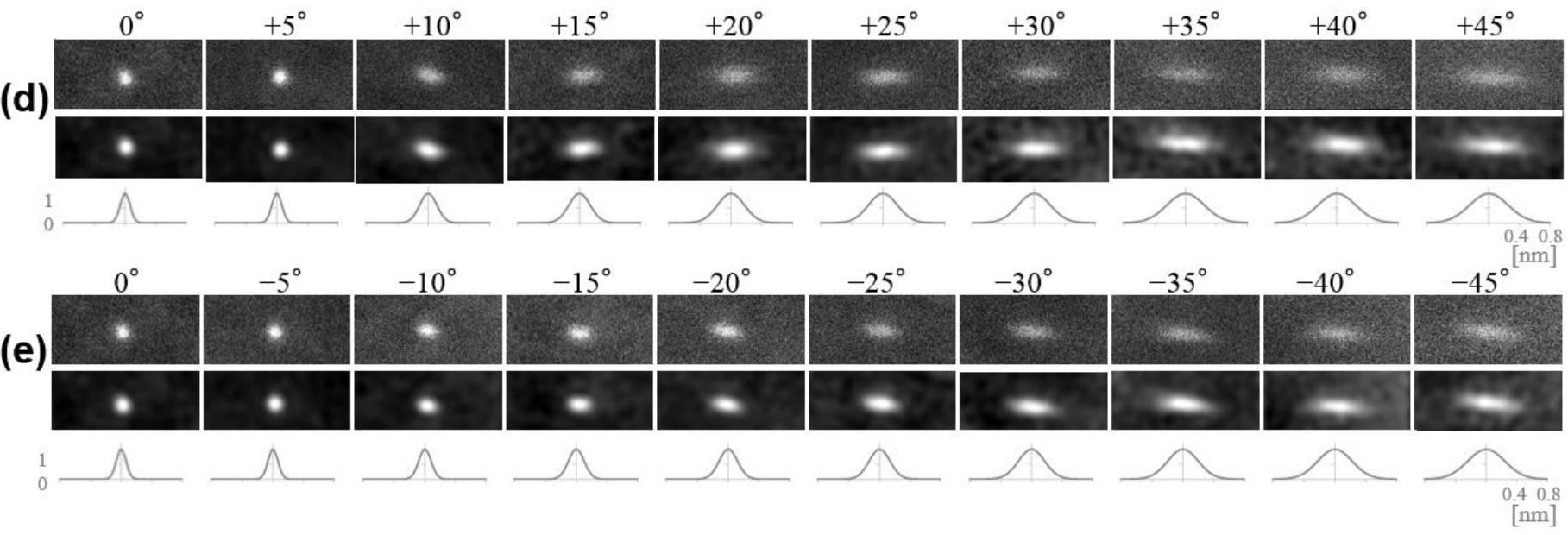

Fig. 2. (a) High resolution HAADF STEM images of gold particles on a carbon film (Fig.S8(b) in Supplementary Material 8) recorded at $80 \mathrm{kV}$ with a specimen tilt of $0^{\circ}$. (b) High resolution HAADF STEM image of gold particles on carbon at $80 \mathrm{kV}$ with a specimen tilt of $45^{\circ}$ along the $\mathrm{x}$ axis. (c) Schematic of the displacement, $\mathrm{z}_{\mathrm{V}}$ along the $\mathrm{z}$ direction and the lateral component, $D_{\mathrm{v}}$. HAADF STEM images recorded with specimen tilt visualize the displacement as a lateral component of $\mathrm{zv}$. (d) Averaged HAADF images of a single atom for tilt angle from $0^{\circ}$ to $+45^{\circ}$ along the $\mathrm{x}$ tilt axis formed from six aligned images of single atoms are displayed in the upper row. Low-pass filtered images of the averaged images with a frequency cutoff of $0.11 \mathrm{~nm}^{-1}$ are displayed in the middle row. Fitted Gaussian functions of the intensity profile along the elongated direction extracted from the low-pass-filtered image are displayed in lower row. (e) Corresponding averaged HAADF images, low-pass filtered images, and the fitted Gaussian functions of a single atom for tilt angle from $0{ }^{\circ}$ to $-45^{\circ}$ along the $\mathrm{x}$ tilt axis. 

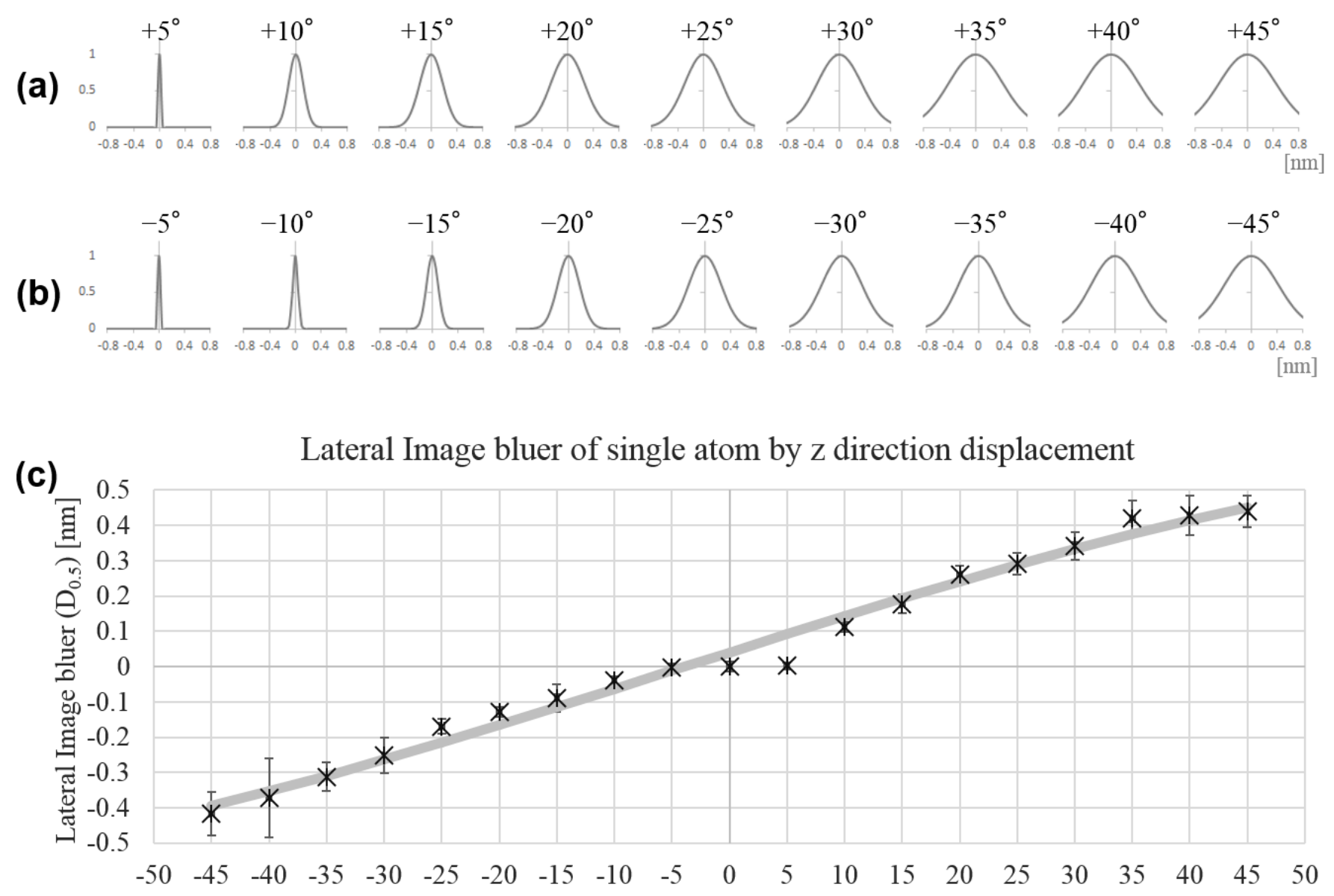

Tilting angle $\left[^{\circ}\right]$

Fig. 3. (a) Gaussian functions for each tilt along the $\mathrm{x}$ axis from $5^{\circ}$ to $45^{\circ}$ de-convolved with the $0^{\circ}$ probe function. This function describes the amplitude of projected component, $\mathrm{z}_{\mathrm{v}}$ in the lateral direction. (b) De-convolved Gaussian functions with for specimen tilt along the $\mathrm{x}$ axis from $-5^{\circ}$ to $-45^{\circ}$ (c) Plots of the lateral image displacement as a function of specimen tilt along the $\mathrm{x}$ axis. The tilt angle was calibrated using a Kikuchi pattern recorded from a crystalline specimen. The average of each $5^{\circ}$ step from $-45^{\circ}$ to $45^{\circ}$ was measured to be $4.99( \pm 0.2)^{\circ}$ [Supplementary Material 10]. The gray solid line describes a fitted function, $\mathrm{D}_{V 0.5}=\mathrm{z}_{V 0.5} \cdot \sin \left(\theta-\theta_{0}\right)$, where $\mathrm{z}_{V 0.5}=0.598 \mathrm{~nm}( \pm 0.15 \mathrm{~nm})$ with $\theta_{0}=3.85^{\circ}$. 